\title{
Designing Interfaces for Presentation of Opinion Diversity
}

Sean A. Munson

School of Information

University of Michigan

1075 Beal Avenue

Ann Arbor, MI 48105 USA

samunson@umich.edu

Daniel Xiaodan Zhou

School of Information

University of Michigan

1075 Beal Avenue

Ann Arbor, MI 48105 USA

mrzhou@umich.edu
Paul Resnick

School of Information

University of Michigan

1075 Beal Avenue

Ann Arbor, MI 48105 USA

presnick@umich.edu

\begin{abstract}
News aggregators rely on links and users votes to select and present subsets of the large quantity of news and opinion items generated each day. Opinion diversity in the output sets can provide several benefits. We outline a range of diversity goals and discuss user reactions to a pilot implementation that selects for diversity as well as popularity. We then describe plans for research on alternative presentations and their impacts on users.
\end{abstract}

\section{Keywords}

Diversity, recommender systems, politics, bias, presentation, blogs, news aggregator

\section{ACM Classification Keywords}

H.5.m Miscellaneous

\section{Introduction}

Observers have raised alarms about increasing political polarization of our society, with opposing groups unable to engage in civil dialogue to find common ground or solutions. Sunstein and others have argued that as people have more choices about their news sources, they will live in echo chambers [15]. Republicans and Democrats read different newspapers and watch different TV news stations. They read different political 
books [8]. Left-leaning and right-leaning blogs rarely link to each other [1], and one study finds that in political blog comments, only $13 \%$ of comments expressed disagreement [6]. If people prefer to avoid hearing challenging views, we may see even greater political fragmentation as people get better and better tools for filtering the news based on their own reactions and reactions of other people like them.

It is not clear, however, that everyone prefers to be exposed to only reinforcing viewpoints. Many participants in online political discussions say that they want and seek out discussions with a diverse range of views [14]. In an online experiment, Garrett found that subjects recruited from the readership of left-wing and right-wing sites were attracted to news stories that reinforced their viewpoints and showed mild aversion to clicking on stories that challenged them. Once they looked at those stories, however, they tended to spend more time reading them [5]. There appear to be many individuals who seek at least some opinion diversity, though they may find it more palatable if also accompanied by some arguments that reinforce their views.

Readers' tolerance for opinion diversity may not be fixed. For example, highlighting the presence of articles that readers are likely to agree with may cause them to be more open to also reading some they disagree with. That might help news aggregators keep readers with minority viewpoints, increasing the diversity of membership and of the opinions expressed in conversations convened around news aggregator results.

\section{Diversity Goals}

Beyond retaining readers with minority viewpoints, there are several societal reasons why some form of diversity might be valuable. One diversity goal is to ensure that everyone is exposed to challenging viewpoints. A long history of experiments has shown that deliberation on an issue with like-minded people leads to polarization: everyone tends to end up with more extreme views than they started with [16]. One explanation for this finding is that, in like-minded groups, people are exposed only to arguments on one side of the issue [4]. Thus, selective exposure to reinforcing news and opinion articles might also lead to opinion shifts to more extreme positions, which would make it harder for society to find a political consensus on important issues. Awareness and inclusion of minority views can also lead to more divergent, out of the box thinking, which can improve individual and group problem solving and decision-making $[7,10,11]$.

A second diversity goal is to make as many people as possible feel that their viewpoints are represented in the aggregator's result set. People who feel that their view is a minority position and so far unspoken may remain silent to promote social harmony $[9,12]$; by making people see that their viewpoints are publicly represented in the selected news and opinion items, people may be more likely to articulate their viewpoints in discussion at the news aggregator site and elsewhere. Moreover, people may be more open to hearing challenging opinions once they feel their own viewpoint is represented [5], so making more people feel included may induce more people to expose themselves to challenging viewpoints.

A third diversity goal is to represent viewpoints in the result set in proportion to their popularity. This could help everyone to understand the relative popularity of different viewpoints. There is a natural tendency for 
people, particularly those in the minority, to think that their own views are more broadly shared than they actually are [13]. Having a better assessment of their true popularity may lead people to accept the legitimacy of disagreeable outcomes in the political sphere, rather than concocting conspiracy theories to explain how the supposed majority will was thwarted.

Developing techniques that allow news aggregators to select diverse sets of items may not be sufficient to achieve desired user reactions. Even if ideas are represented in the news aggregator's results in the same proportion with which they are held, people holding minority opinions may not notice the small number of items representing their views among the many more that challenge their opinions, and they may seek out sources where they can more easily find items that affirm their views. Over time, even people who would prefer to get a mixed selection of news, and to participate in discussions with people who have mixed views, would end up sorting themselves into homogeneous groups. We propose to approach this challenge by conducting a series of experiments on user reactions to collections with different presentations of affirming, challenging, and neutral items.

\section{Initial News and Opinion Aggregator}

To begin exploring the possibilities, we implemented a simple version of an aggregator that selected items based on the number of links they received from blog entries in a collection of 494 political blogs. We coded each of the source blogs based on their political ideology (liberal, independent, or conservative). Coding was based on the researchers' assessment as well as thirdparty classification from Wonkosphere.com and PresidentialWatch08com. Our panel of blogs contained 257 liberal blogs (52\%), 174 conservative blogs (35\%), and 63 independent blogs (13\%).

We then built a web-survey where users viewed the current most popular items. The list showed the top $k$ items selected either based only as a sum of timeweighted votes for the items in the set, or based on a dynamic influence suppression algorithm that reduced the dominance of the (liberal) majority. We describe this algorithm in a companion article. We then asked 39 users to view a set of 12 items (Figure 1 ) generated by one of the two algorithms.

The algorithm itself is not the focus of this article. Comments from subjects who viewed result sets from the algorithms, however, offer insights into the range of individual preferences for diversity. We did not tell subjects that we were studying diversity, but many readers focused on this in their comments. In reporting the comments, we label participants by which algorithm generated their result set; $p$ indicates the popularity algorithm and $s$ indicates the influence suppression algorithm.

Some subjects were happy with the results because of their diversity. "I make a point of visiting websites with

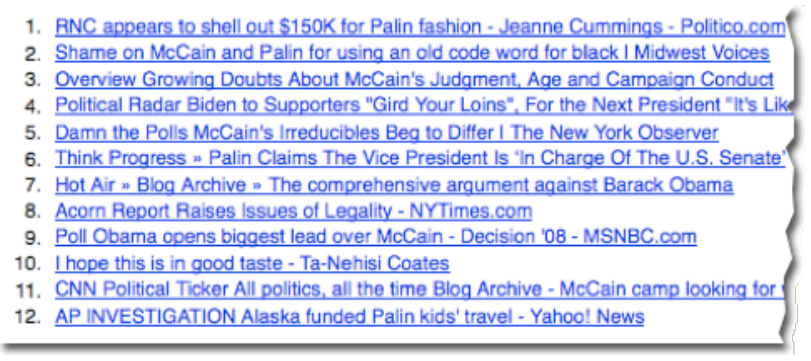

Figure 1. We presented readers with a list of links and page titles and asked for their reactions to the collection of items. 
viewpoints different than my own, so I would have been happy with this" ( $s-12)$. "The aggregator provided a good diversity of viewpoints, [from sites] I don't tend to read" (s-14).

Other users felt that the range of opinion was too close to the center and wanted to see a greater breadth in the result, including views that were less "narrow" ( $\mathrm{s}$ $10 ; p-19)$ or more "strident" (p-36). This was balanced by some who wanted the list to present items that were, on the whole, closer to the center ( $p-17, p-21, p-$ 25).

One reader said that he would not normally use a news aggregator, but liked the results because they were biased in agreement with his political preferences ( $p$ 29 ). Another reader felt that opinions were too challenging: "it's good to know diverse opinions, but, on the other hand, I can't take too much of the opinions that disagree with mine" (p-39).

Users also expressed mixed opinions about the sourcetype diversity. Three mentioned being pleased with the variety of links but eight others found this jarring and would have preferred items from other one type of source (e.g. mainstream media or blogs; $\mathrm{p}-2, \mathrm{p}-3, \mathrm{~s}-$ $11, \mathrm{p}-16)$ or a list that pointed exclusively to either opinion or factual ( $p-7, s-14, s-23)$ items.

Shortcomings of our aggregator unrelated to opinion or source diversity - particularly topic redundancy - also drove satisfaction down in both conditions. Unlike many popular news aggregators (e.g. Google News, Memeorandum), we do not cluster similar articles. On days when one particular news story was receiving sub- stantial attention, notably right around the Presidential election, when most of our subjects completed the survey, the list of 12 items presented to survey respondents might contain many links to different coverage of the story; seven subjects complained about this in the free response section of the survey. Future iterations of our aggregation algorithms - both the baseline we use for comparison and those designed to promote diversity - should cluster related stories by topic and either display them as a cluster or only display the most popular to reduce redundancy in the result sets.

Our prototype aggregator also simply extracts the text in the title tag of each page to use as the link text. In most cases, this contains the article headline and the source, but some page titles are not as descriptive. Better headline extraction is another basic news aggregator feature that we will have to include in order to keep users from being distracted by irrelevant weaknesses in the news aggregator's design.

We also built a basic Google gadget that displays the current news results from the blog link data. Viewers have the option of enabling shading of items according to the mean bias of blogs linking to the item. This highlighting has similarities to a Greasemonkey script Baio and Schachter built to visualize bias on Memeorandum.com [2]. In just a few weeks, over 100 users have installed our web application on their blogs and personal homepages.

\section{Future work}

We plan a series of studies that will allow us to evaluate the effects of the presentation of diversity in result sets on reader reactions. 


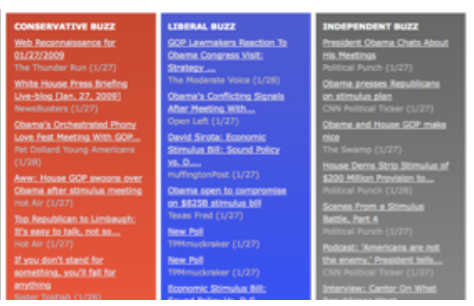

Figure 2. Wonkosphere.com presents results in different lists.

\section{US Pollitical News \& Opinion}

- Caroline Kennedy to Seek Clinton... - ABC News Bush 'Not Insulted' by Thr... - Limits on Executive Pay May Prove T... - Kast sko mot Bush

- Majority of Public Odposes Auto Res... - Washington Wire - WSJ.com The Real...1 - Google Public Policy Blog Net neutr... - Rasmussen Reports 'in The most c. - Jane Hamsher Caroline Kennedy Lets. - Iraais Hail Journalist Who Threw Sh.. - States' Funds for Jobless Are - Rsum Long on Politics but Short on

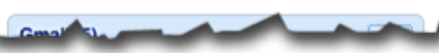

Figure 3. Gadget displaying Sidelines results highlighted according to bias, installed on a user's personalized Google page.
We anticipate that as we vary the number of supporting and challenging items and subjects' assessments of a collection of articles, we will affect whether users feel their opinions are represented in a given result set. A follow-up set of experiments will examine whether alternative presentations moderate these relationships. Swearingen and Sinha have shown that system interface design features influence users' perceptions of collections of recommended products [17], and we anticipate that even with the same result snapshot, changes in the presentation of news aggregator results - such as order of results or color-coding - can affect whether users feel represented or alienated.

Our first presentation experiment will vary the sort order, placing the items predicted to be supportive of the subject's viewpoint at the top of the list, the bottom of the list, or in their original selection order. We expect that subjects will be more satisfied with identical result sets when they are sorted in descending order of expected agreement.

There are many ways that diverse results can be presented. In our pilot, we displayed all items in the same list, without any visual distinction of the different views represented. At the other extreme, used on sites such as Skewz.com and Wonkosphere, separate lists of popular stories for each cluster of views (e.g. Liberal and Conservative) can be built and displayed side-byside (Figure 2).

A second presentation experiment will explore some options between these extremes. We will present items in their original selection order but will color-code them. In one condition, those items that are predicted to be popular among conservatives will be colored red, and those predicted to be popular among liberals will be colored blue, as in the Google gadget that we described in the pilot work section (Figure 3). Because the color-coding can be visually processed more quickly than the text of the links, position may matter less as users are able to tell at a glance whether articles popular with others of their political affiliation are included in the result set. Alternatively, seeing lots of items shaded with the opposing party's color may increase alienation. To assess that, we will also include a condition where especially agreeable items are color-coded but challenging items are not distinguished from neutral items. Using both lab experiments and longitudinal field trials, we will assess users' reactions to different collections of links: how satisfied do they feel with the collections? how represented do they feel?

While color coding or sorting may make people more acceptant of results that include many challenging items, such presentation may also defeat the purpose of presenting diverse results. We will track clickthroughs and the order of clicks to assess the impact of different presentations on the number of supporting and challenging items opened and the time spent reading them. Are users less likely to click through to challenging items if they are highlighted as potentially challenging? Do users first visit items that are predicted to be agreeable?

The field trials will also allow us to measure retention rates, opinion shift, and learning over time based on different presentation techniques. Learning consists of both knowledge of political issues and how widely held different political views are - some presentations of information may better overcome the false consensus effect [13]. 
We expect to identify both optimal practices and tradeoffs. For example, bias highlighting or moving agreeable items to the top of the list may help people in the minority feel more represented by just a few items while also helping people identify and elect not to read potentially challenging items. The first outcome may help retain more diverse users but the second outcome may lead to polarization even with diverse users of a common tool.

\section{Conclusion}

Exposing citizens to a diverse range of opinions and ideas is an important challenge for preserving democracy. Despite the benefits, we cannot force people to seek out and read information that challenges their views. Through research on alternative presentation of items in news aggregators we hope to make diversity palatable or even preferable, even when a user is in the minority, so that people will choose it voluntarily.

\section{References}

[1] Adamic, L and N Glance. The Political Blogosphere and the 2004 US Election: Divided They Blog, Proceedings of the 3rd international workshop on Link Discovery 2005, pp. 36-43.

[2] Baio, A and J Schachter. "Memeorandum Colors: Visualizing Political Bias with Greasemonkey." Waxy.org. 10 October 2008.

[3] Brin, $\mathrm{S}$ and L Page. The anatomy of a large-scale hypertextual Web search engine, Computer Networks and ISDN Systems 30(1), 1998, pp. 107-117.

[4] Burnstein, E and A Vinokur. Persuasive argumentation and social comparison as determinants of attitude polarization. Journal of Experimental Social Psychology 13(4), 1977, pp. 315-332.
[5] Garrett, RK. Exposure to Controversy in an Information Society. Doctoral Dissertation, University of Michigan. 2005. http://hdl.handle.net/2027.42/39374

[6] Gilbert, E, Bergstrom, T and Karahalios, K. Blogs Are Echo Chambers: Blogs Are Echo Chambers. HICSS 2009.

[7] Hong, L and SE Page. Problem Solving by Heterogeneous Agents, Journal of Economic Theory 97(1), 2001, pp. 123-163.

[8] Krebs, V. Political Polarization in Amazon.com Book Purchases. orgnet.com, 2008.

[9] Mansbridge, J. Beyond Adversary Democracy. New York, NY, Basic Books, 1980.

[10] Nemeth, C]. Differential contributions to majority and minority influence. Psychological Review 93(1), 1986, pp. 23-32.

[11] Nemeth, CJ and J Rogers. Dissent and the search for information. British Journal of Social Psychology 35, 1996, pp. 67-76.

[12] Rosenburg, M. Some Determinants of Political Apathy. Public Opinion Quarterly 18 (Winterly), 1955, pp. 349-366.

[13] Sanders, GS and B Mullen. Accuracy in perceptions of consensus: Differential tendencies of people with majority and minority positions. European Journal of Social Psychology 13(1), 1982, pp. 57-70.

[14] Stromer-Galley, J. Diversity of Political Opinion on the Internet: Users' Perspectives. Journal of ComputerMediated Communication 8(3), 2003.

[15] Sunstein, C. Republic.com. Princeton University Press, Princeton, NJ, 2001.

[16] Sunstein, C. The Law of Group Polarization. The Journal of Political Philosophy 10(2), 2002, pp. 175195.

[17] Swearingen, $K$ and $R$ Sinha. Interaction Design for Recommender Systems, Proceedings of DIS2002, London. 